\title{
The Spectrum of Pulmonary Toxicity in Pancreatic Cancer Patients Receiving Gemcitabine Combination Chemotherapy
}

\author{
Jalal Baig ${ }^{\text {a }}$ Mohammad Shokouh-Amiri ${ }^{b}$ Juliana Chan ${ }^{c}$ \\ Rozina Chowdhery ${ }^{a}$ Samaya Danthurthy ${ }^{a}$ Neeta K. Venepalli ${ }^{a}$ \\ aDepartment of Medicine, Division of Hematology/Oncology, University of Illinois at \\ Chicago, Chicago, IL, USA; bepartment of Medicine, Department of Pathology, University \\ of Illinois at Chicago, Chicago, IL, USA; 'College of Pharmacy, University of Illinois at \\ Chicago, Chicago, IL, USA
}

\section{Keywords}

Gemcitabine $\cdot$ Pulmonary toxicity $\cdot$ Combination chemotherapy $\cdot$ Pancreatic cancer

\begin{abstract}
Gemcitabine is widely utilized in the treatment of pancreatic, ovarian, and non small cell lung cancers. Gemcitabine is associated with a wide spectrum of lung toxicities, ranging from dyspnea $25 \%$ of patients to severe pulmonary toxicity in up to $5 \%$ of patients. There is a dearth of information specific to pulmonary toxicity in the setting of gemcitabine combination chemotherapy. Given the potential severity, it is important to identify it early by excluding more common etiologies. We share two case reports of patients with pancreatic cancer who developed severe pulmonary toxicity during gemcitabine combination chemotherapy. Both cases emphasize the heightened risk of pulmonary toxicity in patients receiving gemcitabine chemotherapy combinations, and a need to be vigilant to initiate appropriate therapies immediately.




\section{Case Reports in Oncology}

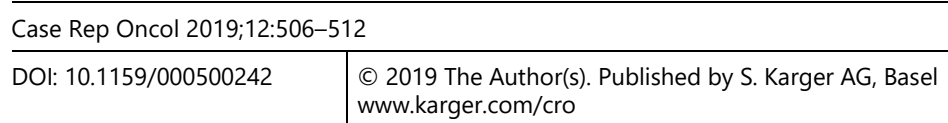

Baig et al.: The Spectrum of Pulmonary Toxicity in Pancreatic Cancer Patients Receiving Gemcitabine Combination Chemotherapy

\section{Introduction}

Gemcitabine is a pyrimidine analog with efficacy in the treatment of many solid tumor malignancies, such as pancreas, ovarian and non-small cell lung cancer (NSCLC) [1]. Pancreatic cancer is a devastating diagnosis with a grim prognosis. Median overall survival ranges from 6-12 months in the metastatic setting [2].

Gemcitabine is associated with improved overall survival (OS) and disease free survival [3] in the metastatic setting (either single agent or in combination with erlotinib or abraxane) and adjuvant setting (OS is increased in combination with capecitabine) [4]. While gemcitabine is generally well tolerated, common toxicities include myelosuppression, peripheral edema, gastrointestinal toxicity and electrolyte abnormalities. Pulmonary toxicities are also common with a $23 \%$ incidence rate, ranging from mild bronchospasm and dyspnea to severe pulmonary toxicity including severe ARDS, pneumonitis, and pulmonary fibrosis [5]. Gemcitabine-induced severe pulmonary toxicity (GISPT) progresses rapidly with mortality rates of $20 \%$ [6] and is associated with variable response even with quick initiation of supportive treatment. We describe below two cases of pulmonary toxicity in the setting of gemcitabine combination chemotherapy. Ultimately, gemcitabine induced pulmonary toxicity is a diagnosis of exclusion, but clinicians should remain vigilant with timely diagnosis and aggressive treatment.

\section{Case Report}

Case 1

A 75-year-old Iranian man without a smoking history was diagnosed incidentally with a pancreatic mass while undergoing evaluation for an abdominal aortic aneurysm. Co-morbidities included hypertension, obstructive sleep apnea, and chronic venous insufficiency. He underwent a Whipple procedure at an outside facility with final pathology confirming pT2N0 (Stage IB) pancreatic ductal adenocarcinoma arising from an intraductal papillary mucinous neoplasm. The patient began adjuvant chemotherapy with gemcitabine $\left(1,000 \mathrm{mg} / \mathrm{m}^{2}\right.$, as $1,862 \mathrm{mg}$ on day 1,8 , and 15 on a 28-day cycle for 6 cycles $)$ and oral capecitabine $\left(620 \mathrm{mg} / \mathrm{m}^{2}\right.$, as 1,150 mg BID on days 1-21). After completion of D1, he presented to the ED on D7 with a nonproductive cough without fevers and with a normal oxygen saturation of $98 \%$. CT chest reported mild diffuse interstitial and parenchymal scarring bilateral lungs, and partial consolidation/atelectasis of the left lung base. He was discharged home with a 7-day course of levofloxacin.

D8 chemotherapy was delayed and given on Day 15, and Day 15 chemotherapy was given Day 22.

Ten days after the completion of the first cycle of chemotherapy, he was taken to the ED for a fall and subsequent dizziness. In the ED, was found to be hypotensive to 90/60 and tachycardic to 111. He was admitted for further workup for syncope and pulmonary embolism. Significant hydration was started for orthostatic hypotension. While evaluation for thrombus was negative, CT chest showed a stable opacity in the left lower lobe and new scattered nodular opacities in bilateral lungs. 
He spiked a fever on day 2 of admission and was started on broad spectrum antibiotics for presumed pneumonia. Fevers remained persistent so antifungal therapy was added on day 4. Also on day 4 of the hospitalization, oxygen level decreased into the low 80s, requiring high flow nasal cannula. ABG showed hypoxemia. CXR revealed bilateral pulmonary edema and patient was transferred to the MICU for diuresis and positive pressure ventilation.

On day 5, patient had PEA arrest with subsequent ROSC after two rounds of chest compressions. He was intubated and started on pressor support. While ECHO was normal, CT scan of the chest showed diffuse bilateral airspace and interstitial opacities with concern for acute respiratory distress syndrome caused by an infectious process (pseudomonas aeruginosa found on sputum culture) and/or pulmonary edema. Due to persistent respiratory failure, IV methylprednisolone $500 \mathrm{mg}$ BID was started on day 8 of hospitalization for presumed gemcitabine-related toxicity.

His respiratory status did not improve despite one week of high dose steroid administration, continued diuresis, and changes to antibiotics. Due to worsening respiratory disease, the family made a decision to extubate and pursues comfort care measures.

On autopsy, bilateral acute bronchopneumonia, secondary diffuse alveolar damage with foci of hyaline membranes, and bilaterally congested and enlarged lungs were found (Fig. 1).

Case 2

A 67-year-old Asian female presented in September 2013 with persistent diarrhea and steatorrhea. A subsequent EGD/EUS revealed a pancreatic head mass. She then underwent a Whipple procedure with pathology notable for papillary mucinous carcinoma with in situ and invasive carcinoma (Fig. 2). Other co-morbidities included hypertension. She did not smoke or drink alcohol.

She received adjuvant gemcitabine $\left(1,000 \mathrm{mg} / \mathrm{m}^{2}\right.$ on day 1,8 , and 15 on a 28 -day cycle for 6 cycles). Follow-up imaging soon after completion of adjuvant therapy showed mesenteric/retroperitoneal/portocaval lymphadenopathy, bilateral lung nodules, and a liver lesion. She then received 24 cycles of palliative FOLFIRINOX with oxaliplatin held intermittently for grade 2 neuropathy. Upon further progression, second-line gemcitabine $1000 \mathrm{mg} / \mathrm{m} 2$ on day 1,8 , and 15 on a 28 -day cycle) and abraxane $\left(125 \mathrm{mg} / \mathrm{m}^{2}\right.$ on day 1,8 , and 15 on a 28 -day cycle) were started. The doses of gemcitabine and abraxane were reduced on cycle two day eight to 800 and $100 \mathrm{mg} / \mathrm{m}^{2}$, respectively, due to thrombocytopenia.

Twelve days after the second cycle was completed, she presented to the hospital with dyspnea, hemoptysis, and fever. ECHO was normal and CT scan of the chest showed bilateral ground glass opacities concerning for alveolar hemorrhage. Pulmonology was consulted for bronchoscopy, which was negative for hemorrhage, malignancy, and infectious etiologies including tuberculosis.

On day 3 of the hospitalization, she became febrile and her oxygen saturation decreased into the 70s. Empiric antibiotics were started for healthcare-associated pneumonia and fevers subsided. Despite this, her respiratory status continued to worsen as oxygen requirements increased to as high as $50 \%$ by ventimask. Crackles were heard on clinical exam and a repeat CT scan showed worsening ground glass opacities and a fibrotic appearance with traction bronchiectasis and septal thickening, which was thought to represent possible drug reaction, pulmonary hemorrhage, ARDS, or viral pneumonia 
After a trial of diuresis failed to improve her oxygenation, IV methylprednisolone $100 \mathrm{mg}$ daily was started for gemcitabine-induced pulmonary toxicity on day 6 . Her oxygen requirement began to improve, and IV antibiotics course was completed. She was discharged with an oral steroid taper and home oxygen to be used on exertion.

\section{Discussion}

Gemcitabine-induced lung toxicity is a diagnosis of exclusion. It can range from mild dyspnea and bronchospasm to severe dyspnea, diffuse alveolar damage, acute respiratory distress syndrome (ARDS), interstitial pneumonitis or noncardiogenic pulmonary edema [2]. While the mechanism of gemcitabine-induced lung toxicity remains unclear, contributing factors include increased capillary permeability resulting in pulmonary edema, and a cytokine-based inflammatory reaction involving tumor necrosis factor alpha or various interleukins contributing to ARDS via an interstitial inflammatory process [7].

GISPT has been documented in persons with lung, breast, pancreas and Hodgkin disease, happening at a median interval of 48 days post initiation of gemcitabine [8]. Onset has been noted even one day after initiation of therapy, with rare cases reported of late-onset toxicity [6].

Risk factors include previous pulmonary disease, concomitant administration of bleomycin, chemotherapy agents known to release cytokine mediators of inflammation such as vinorelbine, paclitaxel, or docetaxel, and concomitant use of thoracic irradiation. Whether combination chemotherapy potentiates lung toxicity is unclear (Table 1); combination chemotherapy pulmonary toxicity is estimated at 10-43\% [8], with higher incidence of pulmonary toxicity found in gemcitabine in combination with vinorelbine, pacelitaxel, docetaxel [8] and gemcitabine in combination with S1 or erlotinib [9]. Gemcitabine-induced cytokine release may exacerbate the pulmonary toxicity of coadministered drugs. Additionally, coadministered drugs may compound gemcitabine-induced cytokine release or thwart the anti-inflammatory counter response to gemcitabine-triggered cytokine release [8].

A diagnosis of chemotherapy-induced pulmonary toxicity can be made when symptoms develop shortly after the onset of therapy and an alternative respiratory or cardiac diagnosis is not found despite a thorough evaluation. GISPT is a diagnosis of exclusion after pneumonia, pulmonary embolus, cardiogenic pulmonary edema, malignancy, diffuse alveolar hemorrhage and exacerbation of chronic lung conditions are not found [10]. Once it is diagnosed, the drug must be discontinued and corticosteroids, continuous or pulse dose, and appropriate pulmonary support must be initiated. While a rapid response to steroids can be seen, the mortality rate can be as high as $20 \%$ [11].

Both patients above experienced new onset pulmonary toxicities while receiving gemcitabine combination chemotherapy, however the first patient's case was confounded by fevers, pneumonia, and ARDS in the setting of profound sepsis. Additionally, the first patient did not improve despite high dose steroid administration. We concluded that while the first case is possible GISTP, the second case is probably GISTP given response to steroids. Additionally, although literature suggests additional pulmonary toxicity with combination gemcitabine and capecitabine [12], to our knowledge, this is the first case report to date reporting on severe pulmonary toxicity for gemcitabine-capecitabine in the adjuvant setting. Regardless of the 
total duration of gemcitabine therapy received, physicians should be suspicious of drug-induced pulmonary toxicity whenever respiratory distress is evaluated in a patient receiving gemcitabine chemotherapy combinations.

\section{Conclusion}

The cases presented above reinforce the variable onset of gemcitabine-induced lung toxicity with the first patient presenting within one week of completing cycle 1 and the second patient presenting after cycle 2 . There is a heightened risk for a spectrum of lung toxicities in pancreatic cancer patients receiving gemcitabine chemotherapy combinations. Thus, there should be a lower threshold to stop therapy and initiate steroids promptly if any pulmonary symptoms appear suspicious.

\section{Acknowledgements}

The authors would like to acknowledge the family members of the patients above.

\section{Statement of Ethics}

The families of the patients have given their written informed consent to publish their case.

\section{Disclosure Statement}

The authors have no conflicts of interest to declare.

\section{Funding Sources}

The authors have no funding sources.

\section{Author Contributions}

NKV, RC, MS, JC, JB, SD conceived and designed the case report. MS contributed pathology images and pathology review. NKV, JB, SD, JC, MS were involved in drafting and revising the manuscript. All authors read and approved the final manuscript. 
Baig et al.: The Spectrum of Pulmonary Toxicity in Pancreatic Cancer Patients Receiving Gemcitabine Combination Chemotherapy

\section{References}

1 Gupta N, Ahmed I, Steinberg H, Patel D, Nissel-Horowitz S, Mehrotra B. Gemcitabine-induced pulmonary toxicity: case report and review of the literature. Am J Clin Oncol. 2002 Feb;25(1):96-100.

2 Chi DC, Brogan F, Turenne I, Zelonis S, Schwartz L, Saif MW. Gemcitabine-induced pulmonary toxicity. Anticancer Res. 2012 Sep;32(9):4147-9.

3 Oettle H, Neuhaus P, Hochhaus A, Hartmann JT, Gellert K, Ridwelski K, et al. Adjuvant chemotherapy with gemcitabine and long-term outcomes among patients with resected pancreatic cancer: the CONKO-001 randomized trial. JAMA. 2013 Oct;310(14):1473-81.

4 Neoptolemos JP, Palmer DH, Ghaneh P, Psarelli EE, Valle JW, Halloran CM, et al.; European Study Group for Pancreatic Cancer. Comparison of adjuvant gemcitabine and capecitabine with gemcitabine monotherapy in patients with resected pancreatic cancer (ESPAC-4): a multicentre, open-label, randomised, phase 3 trial. Lancet. 2017 Mar;389(10073):1011-24.

5 Barlési F, Villani P, Doddoli C, Gimenez C, Kleisbauer JP. Gemcitabine-induced severe pulmonary toxicity. Fundam Clin Pharmacol. 2004 Feb;18(1):85-91.

6 Sherrod AM, Brufsky A, Puhalla S. A case of late-onset gemcitabine lung toxicity. Clin Med Insights Oncol. 2011;5:171-6.

7 Ko E, Lee S, Goodman A. Gemcitabine pulmonary toxicity in ovarian cancer. Oncologist. 2008 Jul;13(7): 807-11.

8 Belknap SM, Kuzel TM, Yarnold PR, Slimack N, Lyons EA, Raisch DW, et al. Clinical features and correlates of gemcitabine-associated lung injury: findings from the RADAR project. Cancer. 2006 May;106(9):2051-7.

9 Tamiya A, Endo M, Shukuya T, Igawa S, Tsuya A, Nakamura Y, et al. Features of gemcitabine-related severe pulmonary toxicity: patients with pancreatic or biliary tract cancer. Pancreas. 2009 0ct;38(7):838-40.

10 Shaib W, Lansigan F, Cornfeld D, Syrigos K, Saif MW. Gemcitabine-induced pulmonary toxicity during adjuvant therapy in a patient with pancreatic cancer. JOP. 2008 Nov;9(6):708-14.

11 Vahid B, Marik PE. Pulmonary complications of novel antineoplastic agents for solid tumors. Chest. 2008 Feb;133(2):528-38.

12 Chan AK, Choo BA, Glaholm J. Pulmonary toxicity with oxaliplatin and capecitabine/5-Fluorouracil chemotherapy: a case report and review of the literature. Onkologie. 2011;34(8-9):443-6.

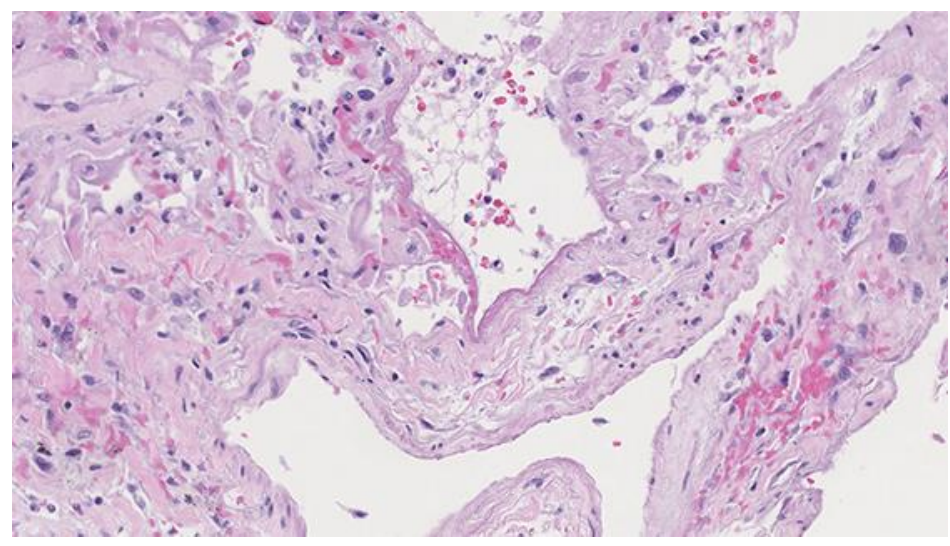

Fig. 1. Lung, left upper lobe, showing diffuse alveolar damage with foci of hyaline membrane formation. 


\section{Case Reports in Oncology}

Baig et al.: The Spectrum of Pulmonary Toxicity in Pancreatic Cancer Patients Receiving Gemcitabine Combination Chemotherapy

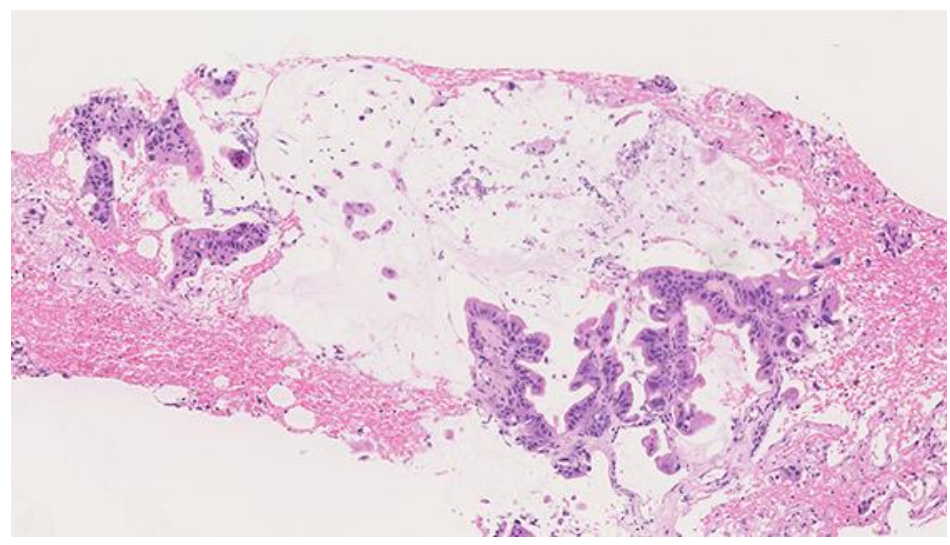

Fig. 2. Lung, right upper lobe, biopsy, metastatic mucinous adenocarcinoma, with primary pancreatic carcinoma.

Table 1. Case reports of pulmonary toxicity in stage IV pancreatic cancer patients on gemcitabine combination chemotherapy regimens

\begin{tabular}{|c|c|c|c|c|c|}
\hline $\begin{array}{l}\text { Chemotherapy } \\
\text { combination }\end{array}$ & $\begin{array}{l}\text { Age, years/ } \\
\text { Gender }\end{array}$ & $\begin{array}{l}\text { Toxicity onset } \\
\text { post initiation }\end{array}$ & $\begin{array}{l}\text { Pulmonary toxicities } \\
\text { (evaluation) }\end{array}$ & $\begin{array}{l}\text { Treatment pulmonary } \\
\text { toxicities }\end{array}$ & $\begin{array}{l}\text { Outcome pulmonary } \\
\text { toxicity treatment }\end{array}$ \\
\hline $\begin{array}{l}\text { Gemcitabine + } \\
\text { Erlotinib }\end{array}$ & $\begin{array}{l}57 / \mathrm{M}(13) \\
63 / \mathrm{F} \mathrm{(14)} \\
55 / \mathrm{M}(15)\end{array}$ & $\begin{array}{l}7 \text { weeks } \\
6 \text { months } \\
2 \text { weeks }\end{array}$ & $\begin{array}{l}\text { Dyspnea, hypoxia, ILD (CT) } \\
\text { Dyspnea, hypoxia, COP (BAL) } \\
\text { Dyspnea, hypoxia, ILD (CT) }\end{array}$ & $\begin{array}{l}\text { Prednisolone } 900 \mathrm{mg} \text { QD } \\
\times 3 \text { days, } 16 \text { day taper } \\
\text { Steroid taper, not specified } \\
\text { Prednisolone } 1,000 \mathrm{mg} Q D \\
\times 3 \text { days, } 10 \text { week taper }\end{array}$ & $\begin{array}{l}\text { Complete recovery per CT } \\
7 \text { days post steroid initiation } \\
\text { Complete recovery } \\
\text { Complete recovery per CT } \\
14 \text { days post steroid initiation }\end{array}$ \\
\hline $\begin{array}{l}\text { Gemcitabine + } \\
\text { Abraxane }\end{array}$ & $\begin{array}{l}75(16) \\
70 / \mathrm{M} \\
60 / \mathrm{F} \\
53 / \mathrm{F} \\
56 / \mathrm{M}\end{array}$ & $\begin{array}{l}2 \text { months } \\
2 \text { months } \\
3 \text { months } \\
2 \text { months } \\
3 \text { months }\end{array}$ & $\begin{array}{l}\text { Fever, ILD (CT, BAL) } \\
\text { Cough, ILD (CT, BAL) } \\
\text { Fever, Cough (CT, BAL) } \\
\text { Dyspnea (CT, BAL) } \\
\text { Dyspnea (CT, BAL) }\end{array}$ & Variable & Complete recovery \\
\hline $\begin{array}{l}\text { Gemcitabine + } \\
\text { Capecitabine }\end{array}$ & $\begin{array}{l}78 / \mathrm{F}(17) \\
74 / \mathrm{M} \\
73 / \mathrm{M}\end{array}$ & $\begin{array}{l}2 \text { months } \\
2 \text { months } \\
3 \text { months } \\
2 \text { months } \\
3 \text { months }\end{array}$ & Not specified & Not specified & Complete recovery \\
\hline
\end{tabular}

ILD, interstitial lung disease; M, male; F, female; COP, cryptogenic organizing pneumonia; BAL, bronchoalveolar lavage. 